\title{
Clinical and epidemiological differences and risk factors for death among solid organ transplant recipients with Gram-positive and Gram-negative bloodstream infections
}

\author{
Thiago Zinsly Sampaio Camargo ${ }^{1,2^{*}}$, M. Silva Jr${ }^{1}$, A. R. Marra ${ }^{1}$ and L. F. A. Camargo ${ }^{1}$
}

\begin{abstract}
Objectives: Describe clinical and epidemiological differences and risk factors for death among Bloodstream Infections (BSI) caused by Gram Negative (GN) and Gram Positive (GP) bacteria in the setting of solid organ transplantation.

Methods: We performed a retrospective analysis of medical records, which were evaluated patients undergoing solid organ transplantation with BSI in the period from January 2000 to January 31, 2006 at Hospital São Paulo and Hospital do Rim e Hipertensão (Universidade Federal de São Paulo, Brazil). It was also performed the analysis of risk factors for death.

Results: 195 patients were included in this study with a mean age of $43.3( \pm 0.90)$ years, $114(58.5 \%)$ were male and, 81 (41.5\%) were female. 168 (86.2\%) were kidney transplants, 16 (8.2\%) kidney-pancreas, 5 (2.6\%) heart, 5 (2.6\%) liver and $1(0.5 \%)$ liver-kidney. The mean hospital stay was 34.2 ( \pm 62.7$)$ days. GN accounted for 147 (75.4\%) of the BSI episodes, whereas 48 (24.6\%) were caused by GP. In the group with BSI by GN the most common site of infection was the urinary tract in $68(46.3 \%)$ cases, and in the group with BSI by GP the most common was the primary source in 14 (29.1\%) of the cases. The overall mortality of patients with BSI by GN was $19.7 \%$ (29 cases) and by GP was $35.4 \%$ (17 cases) ( $p=0.03)$. In multiple logistic regression analysis, the variables associated independently with death in patients with BSI by GN were those who developed respiratory failure requiring mechanical ventilation Odds Ratio (OR) 13,2 $95 \%$ Confidence Interval $(C l)=3.07-57.19(p=0.001)$, beyond those which had number equal to or greater than two comorbidities OR $12.495 \% \mathrm{Cl}=1.90-80.35(p=0.008)$. In the population with BSI by GP only the respiratory failure requiring mechanical ventilation $\mathrm{OR} 28.3 \mathrm{Cl} 95=2.53-317.1(p=0.007)$ was independently associated with death.
\end{abstract}

Conclusions: Patients with BSI showed the urinary source as the main site of infection. Death within 30 days occurred in 29 (19.7\%) patients with GN and 7 (35.4\%) patients with GP BSI ( $p=0.03)$. Respiratory failure was a risk factor for death in patients with BSI by GN and GP.

\section{Background}

Transplantation is the treatment of choice for many patients with end-stage organ failure from a variety of causes resulting in prolonged survival and better quality of life for patients $[1,2]$.

Among the infectious complications of solid organ transplantation, bacteremia is a major cause of mortality [3]. Its incidence is estimated at 28 to $30 \%$ in liver, 5 to $11 \%$ in kidney, and $11 \%$ in heart transplant recipients
$[4,5]$. Aerobic gram-positive and gram-negative bacteria are the most frequent agents, with variable frequencies depending on the type of transplantation [6]. Mortality rates range from $11-33 \%$, with higher rates occurring among heart transplant patients [7].

Little is known about the differences between bacteremia from gram-negative and gram-positive bacteria in the setting of solid organ transplantation and their specific impact on mortality. This retrospective study aims to evaluate these two issues.

\footnotetext{
* Correspondence: zinsly@terra.com.br

${ }^{1}$ Universidade Federal de São Paulo, São Paulo, Brazil

${ }^{2}$ Hospital Israelita Albert Einstein, São Paulo, Brazil
} 


\section{Methods}

The study was conducted at Hospital São Paulo and Hospital do Rim e Hipertensão, both affiliated to the Federal University of Sao Paulo, Brazil, and assessed a retrospective cohort of solid organ transplant recipients with bloodstream infections (BSIs) in the period from 1 January 2000 to 31 January 2006.

We evaluated only patients over 18 years of age with monomicrobial BSI. In the case of more than one BSI for the same patient, only the first episode was considered. Cases were selected form the microbioloy laboratory reports and CDC criteria was used to classify BSI episodes, excluding false positive infections, and the clinical and epidemiological data were collected from their medical records.

The samples were processed with the BACTEC 9240 (Becton Dickinson, Maryland, USA) system. Biochemical tests for identification and antimicrobial susceptibility testing by disk diffusion were performed according to standard procedures from the Clinical and Laboratory Standards Institute [8].

The variables analyzed were gender, age, type of dialysis, transfusion, immunosuppression (cyclosporine, azathioprine, mycophenolate mofetil, tacrolimus, sirolimus, prednisone, thymoglobulin or interleukin-2 blocker), living or deceased donor, prior treatment for cytomegalovirus infection, acute rejection and its management, Charlson index, length of hospital stay, mechanical ventilation, shock, and the clinical outcome in the hospital.

\section{Statistical analysis}

To evaluate risk factors for mortality, we used a nested case control studies comparing living and deceased patient after BSI. Univariate analysis of risk factors represented by nominal categorical variables was performed using the chi-squared $\left(\mathrm{X}^{2}\right)$ or Fisher's exact test (FET) as appropriate. For continuous variables with normal distribution we used the Student $t$ test and for non-normal distribution we used the Mann-Whitney test. All significance probabilities presented were two-tailed and a p-value of less than 0.05 was considered statistically significant. The odds ratio and its corresponding $95 \%$ confidence interval were analyzed. Variables found to be significant by univariate analysis were then entered into a multivariate model. All statistical analyses were performed using the Statistical Package for the Social Sciences software (version 16.0, SPSS Inc., Chicago, IL).

\section{Results}

We studied 195 patients with bloodstream infection (BSI). Of these, $24.6 \%(n=48)$ had gram-positive (GP $\mathrm{BSI})$ and $75.4 \%(n=147)$ had gram-negative (GN BSI) bloodstream infections $(p=0.94)$. Escherichia coli was isolated in $36.9 \%$ of the patients, and Klebsiella pneumoniae was the second most frequently isolated agent, accounting for $11.8 \%$ of BSI cases. Regarding gram-positive agents, Staphylococcus aureus was the most frequently found agent, isolated in 16 patients, followed by coagulase-negative Staphylococci, found in 15 patients. Regarding the overall distribution of agents, Staphylococcus aureus was the 5th (8.2 \%) and coagulase-negative Staphylococcus was the 6th (7.7\%) most frequent agent (Table 1).

Most patients were males, $57.8 \%$ of the patients with GN BSI and $60.4 \%$ of those with GP BSI $(p=0.75)$. The mean and median ages of patients with GN BSI were respectively 43.61 and 45.00 years, while in the GP BSI group these were 42.31 and 40.50 years. A single transplant was performed in $89.1 \%$ of patients with GP BSI and in $91.7 \%$ of those with GN BSI $(p=0.61)$. Regarding length of hospital stay, the mean and median values were 35.4 days and 18.0 days, respectively, in the group with GN BSI and 30.6 and 25.5 days in the GP BSI group (Table 2).

Of the cases with GN BSI, 129 (87.8 \%) were kidney transplants, $12(8.2 \%)$ were pancreas-kidney, 1 (0.7 \%) was a heart transplant, $4(2.7 \%)$ were liver transplants and $1(0.7 \%)$ was a liver-kidney transplant. Of the cases with GP BSI, 39 (81.3\%) were kidney transplants, 4 (8.3\%) were kidney-pancreas transplants, 4 (8.3\%) were heart transplants, and $1(2.1 \%)$ was a liver transplant (Table 2).

Table 1 Analysis of 195 isolated pathogens in solid organ transplant recipients with bloodstream infection by gram positive and gram negative bacteria

\begin{tabular}{lcl}
\hline Pathogen & Number & Percent \\
\hline Escherichia coli & 60 & 36.9 \\
Klebsiella pneumoniae & 23 & 11.8 \\
Pseudômonas aeruginosa & 20 & 10.3 \\
Enterobacter aerogenes & 20 & 10.3 \\
Staphylococcus aureus & 16 & 8.2 \\
Coagulase negative Staphylococci & 15 & 7.7 \\
Enterococcus spp & 11 & 5.6 \\
Acinetobacter baumannii & 10 & 5.1 \\
Serratia marcescens & 5 & 2.6 \\
Streptococcus pneumoniae & 4 & 2.1 \\
Stenotrophomonas maltophilia & 3 & 1.5 \\
Salmonella spp & 2 & 1.0 \\
Providencia spp & 2 & 1.0 \\
Burkholderia cepacea & 1 & 0.5 \\
Citrobacter spp & 1 & 0.5 \\
Listeria sp. & 1 & 0.5 \\
Streptococcus viridans & 1 & 0.5 \\
\hline
\end{tabular}


Table 2 Univariate and multivariate analysis of risk factors for death among 147 solid organ transplant recipients with bloodstream infection by gram negative bacteria

\begin{tabular}{|c|c|c|c|c|c|c|c|c|}
\hline \multirow[t]{3}{*}{ Variables } & \multicolumn{2}{|c|}{ Cases (147) } & \multicolumn{3}{|c|}{ Univariate } & \multicolumn{3}{|c|}{ Multivariate } \\
\hline & Death & Survival & $\mathrm{OR}$ & IC95 \% & $p$ & $\mathrm{OR}$ & IC95 \% & $p$ \\
\hline & N (\%) & N (\%) & & & & & & \\
\hline Male gender & $20(23.5)$ & $65(76.5)$ & 1.81 & $0.76-4.30$ & 0.21 & & & \\
\hline ICU & $11(52.4)$ & $10(47.6)$ & 6.6 & $2.45-17.78$ & $<0.001$ & 2.10 & $0.54-8.13$ & 0.29 \\
\hline HAls & $15(25.0)$ & $45(75.0)$ & 1.74 & $0.77-3.94$ & 0.21 & & & \\
\hline Charlson $\geq 3$ & $15(31.3)$ & $33(68.7)$ & 2.76 & $1.20-6.34$ & 0.03 & 0.68 & $0.17-2.74$ & 0.59 \\
\hline Co-morb (liver disease) & $5(71.4)$ & $2(28.6)$ & 12.1 & $2.21-65.99$ & 0.003 & 1.45 & $0.13-15.58$ & 0.76 \\
\hline Co-morb (cancer) & $3(75.0)$ & $1(25.0)$ & 13.5 & $1.35-135.0$ & 0.02 & 6.87 & $0.40-118.8$ & 0.18 \\
\hline Co-morbidities ( $\geq 2$ ) & $8(66.7)$ & $4(33.3)$ & 10.9 & $3.00-39.34$ & $<0.01$ & 12.4 & $1.90-80.35$ & 0.008 \\
\hline Deceased donor & $20(24.1)$ & $63(75.9)$ & 1.94 & $0.82-4.61$ & 0.15 & & & \\
\hline Induction therapy & $7(22.6)$ & $24(77.4)$ & 1.25 & $0.48-3.26$ & 0.62 & & & \\
\hline Acute rejection (AR) & $21(48.8)$ & $22(51.2)$ & 1.66 & $0.54-4.24$ & 0.31 & & & \\
\hline AR-corticosteroids & $6(30.0)$ & $14(70.0)$ & 1.94 & $0.67-5.58$ & 0.23 & & & \\
\hline OB-MMF & $5(11.6)$ & $38(88.4)$ & 0.44 & $0.15-1.24$ & 0.17 & & & \\
\hline OB-Ciclosporine & $16(16.8)$ & 79 (83.2) & 0.61 & $0.27-1.39$ & 0.28 & & & \\
\hline Double-J & $4(9.8)$ & $37(90.2)$ & 0.35 & $0.11-1.08$ & 0.07 & & & \\
\hline Pre tx dialysis & $22(17.1)$ & $107(82.9)$ & 0.32 & $0.11-0.93$ & 0.05 & 0.58 & $0.13-2.52$ & 0.47 \\
\hline CMV & $4(20.0)$ & $16(80.0)$ & 1.02 & $0.31-3.32$ & 1.00 & & & \\
\hline Shock & $6(46.2)$ & $7(53.8)$ & 4.14 & $1.27-13.45$ & 0.02 & 0.47 & $0.08-2.65$ & 0.40 \\
\hline Respiratory failure & $15(62.5)$ & $9(37.5)$ & 13.0 & $4.79-35.14$ & $<0.001$ & 13.2 & $3.07-57.19$ & 0.001 \\
\hline Pre tx diabetes & $2(13.3)$ & $13(86.7)$ & 0.60 & $0.13-2.81$ & 0.74 & & & \\
\hline Inadequate atb & $12(26.7)$ & $33(73.3)$ & 1.82 & $0.78-4.21$ & 0.18 & & & \\
\hline
\end{tabular}

ICU Intensive care unit, HAls Health-acquired infections, $O B$ onset of BSI, $T X$ transplant, atb antimicrobial

The organs were transplanted from deceased donors in $83(56.5 \%)$ of the patients with GN BSI and in 23 (47.9\%) of those with GP BSI $(p=0.30)$. Pre-transplant dialysis was required by $129(87.8 \%)$ patients in the GN BSI group and $41(85.4 \%)$ patients in the GP BSI group $(p=0.067)$.

Considering both groups together, the most common primary site of infection was the urinary tract, accounting for $71(36.4 \%)$ cases. BSI ranked second, having been reported as the primary infection in $37(19.0 \%)$ cases, followed by gastrointestinal in $27(13.8 \%)$, pulmonary in 24 (12.3\%), other sites in 23 (11.8\%), surgical wound infection in 7 (3.6 \%) and skin infection in $6(3.1 \%)$ cases.

A double-J stent was used in 41 (27.9\%) cases with GN BSI, while only one (2.1\%) case with GP BSI used this type of device $(p=0.0002)$. A urinary fistula was found in 4 (7.2\%) cases with GN BSI but in none of the patients with GP BSI $(p=0.25)$.

Regarding the clinical features of the different groups studied, 118 (80.3 \%) patients with GN BSI and $40(83.3 \%)$ with GP BSI had Charlson scores of $0-3(p=0.64)$. At the onset of the BSI, $21(14.3 \%)$ patients in the GN group and 7 (14.6\%) in the GP group were hospitalized in the intensive care unit $(p=0.96)$.

Bacteremias were considered as healthcare-associated infections (HAIs) in $60(40.8 \%)$ patients with GN and 19 (39.6 \%) with GP $(p=0.88)$. Post-transplant dialysis was necessary in $51(34.7 \%)$ patients in the GN group and $11(22.9 \%)$ in the GP group $(p=0.13)$. Pretransplant diabetes mellitus was found in $15(10.2 \%)$ patients with GN BSI and in $6(12.5 \%)$ patients with GP BSI $(p=0.66)$, whereas post-transplant diabetes mellitus was found in $19(12.9 \%)$ patients with GN BSI and in 3 $(6.3 \%)$ patients with GN BSI $(p=0.20)$.

Of all patients with BSI, 26 (17.7 \%) in the GN group and $9(18.8 \%)$ in the GP group developed septic shock $(p=0.87)$. Mechanical ventilation was required by 24 (16.3\%) patients with GN BSI and by 14 (29.2 \%) patients with GP BSI $(p=0.052)$.

An indwelling urinary catheter was present in 36 (24.5\%) patients with GN BSI and 15 (31.2 \%) patients with GP BSI $(p=0.36)$. Antibiotic therapy was considered adequate in $102(69.4 \%)$ patients with GN BSI and $32(66.8 \%)$ patients with GP BSI $(p=0.72)$. 
Death within 30 days occurred in 29 (19.7 \%) patients with GN BSI and 7 (35.4\%) patients with GP BSI $(p=0.03)$.

With regard to the immunosuppression regimen used at the time of the BSI onset, prednisone was the drug used in $143(97.3 \%)$ of the patients with GN and in 47 $(97.9 \%)$ of the patients with GP infections $(p=0.81)$. With regard to cyclosporine, 95 (64.6\%) patients used this immunosuppressant in the GN group versus 34 $(70.8 \%)$ in the GP group $(p=0.43)$. Azathioprine was used in $75(51.0 \%)$ patients in the GN group and 24 $(50.0 \%)$ in the GP group $(p=0.90)$. mycophenolate mofetil was used in $43(29.3 \%)$ patients in the first group versus $9(18.7 \%)$ in the second $(p=0.15)$ tacrolimus was used in $36(24.5 \%)$ patients with GN and $6(12.5 \%)$ with GP $(p=0.08)$ and, finally, rapamycin was used in 4 $(2.7 \%)$ patients in the first group and $2(4.2 \%)$ in the second $(p=0.61)$.

In the group with GN BSI, 31 (21.1\%) patients received induction therapy versus $5(10.4 \%)$ in the GP group $(p=0.09)$. The drug chosen for this purpose in 14 $(9.5 \%)$ cases in the first group and in $2(4.2 \%)$ cases in the second group was thymoglobulin $(p=0.24)$. OKT3 was used in $4(2.7 \%)$ patients with GN BSI and in 2 $(4.2 \%)$ patients with GP BSI $(p=0.61)$, whereas the interleukin-2 inhibitor was used in $5(3.4 \%)$ patients in the first group and $1(2.1 \%)$ in the second $(p=0.64)$.

Acute rejection was diagnosed in 30 (20.4\%) of the patients with GN BSI and in 19 (39.6\%) patients with GP BSI $(p=0.008)$. This acute rejection was treated with methylprednisolone in 20 (13.6\%) patients in the first group and $16(37.5 \%)$ in the second $(p=0.003)$. Thymoglobulin was the preferred therapy in $4(7.2 \%)$ patients in the GN group and 1 in the GP group $(p=0.81)$. OKT3 was used in $1(0.7 \%)$ patient in the GN group and 1 $(2.1 \%)$ in the GP group $(p=0.40)$. The interleukin-2 inhibitor was used in $4(7.2 \%)$ patients in the first group and $1(2.1 \%)$ in the second group ( $p=0.81)$.

CMV disease was diagnosed and treated with gancyclovir prior to the BSI onset in $20(13.6 \%)$ patients with GN infections and $3(6.3 \%)$ with GP infections $(p=0.17)$.

A univariate analysis was performed to identify risk factors for death among transplant recipients with BSI, comparing those who died with those who survived.

In the case of GN BSI, risk factors for death were the stay in the ICU at the onset of bacteremia (OR $6.695 \%$ CI 2.45-17.78), a Charlson index $>3$ (OR $2.7695 \%$ CI 1:20-6.34) ( $p=0.03)$, in addition to liver disease (OR $12.195 \%$ CI 2.21-65.99) $(p=0.003)$ and malignancy (OR $5.1395 \%$ CI 1.35-135.0) $(p=0.02)$ as comorbidities, as well as the presence of two or more comorbidities (OR $9.10 \quad 95 \%$ CI $3.00-9.34$ ) ( $\mathrm{p}<0.01$ ) compared with those with one comorbidity or less altogether.
Pre-transplant dialysis (OR $0.3295 \%$ CI $0.11-0.93$ ) $(p=0.05)$ was listed as a protective factor against death, while shock at the onset of bacteremia (OR $4.1495 \% \mathrm{CI} 1.27-13.45)(p=0.02)$ and respiratory failure (OR $13.095 \%$ CI 4.79-35.14) ( $<<0.001)$ were associated with higher mortality rates in this population, based on the univariate analysis (Table 2).

On multiple logistic regression, the variables that were independently associated with progression to death were the development of respiratory failure requiring mechanical ventilation (OR $2.1395 \% \mathrm{CI}$ 3.07-57.19) ( $\mathrm{p}<0.001)$ and $\geq 2$ comorbidities (OR 12.4 $95 \%$ CI 1.90-80.35) $(P=0.008)$ (Table 2).

However, when we analyzed patients with GP BSI, we observed that mortality was higher among those with shock at the onset of bacteremia (OR 16.4 $95 \%$ CI $1.76-151.7)(p=0.005)$ and respiratory failure (OR 34.8 $95 \%$ CI 5.91-204-8) $(\mathrm{p}<0.001)$ based on the univariate analysis. The use of cyclosporine at the onset of bacteremia appeared to be a protective factor against death (OR $0.1795 \%$ CI 0.04-0.66) ( $p=0.02)$ (Table 3).

On multiple logistic regression in this population, the variable that was independently associated with death was the development of respiratory failure requiring mechanical ventilation (OR $28.3 \quad 95 \%$ CI 2.53-317.1) $(p=0.07)$ (Table 3).

Antimicrobial adequacy was not associated to mortality on the univariate analysis for both, GN BSI (OR 1.82 $95 \%$ CI $0.78-4.21)(p=0.18)$, and GP BSI (OR 1.14 $95 \%$ CI 0.33-4.00) ( $p=1.00)$ (Tables 2 and 3).

\section{Discussion and conclusions}

In our study, most of the isolates were gram-negative (75.4 \%), which may be related to the higher number of kidney transplants in our series $(86.2 \%)$; this fact had already been observed by other authors, since the urinary tract is a major source of bacteremia caused by these agents in this population [6]. At the same time, studies concerning liver transplantation have also found a similar microbial distribution, which is assigned to the abdominal source of these infections-the second most common site in our study [9].

In a recent national multicenter study that covered almost three years of epidemiological surveillance of nosocomial bloodstream infections, not only in transplant recipients (the Brazilian SCOPE study), gram-negative agents were also prominent and represented $58.5 \%$ of the isolates [10].

In some European studies that also included different types of solid organ transplantations, gram-positive agents have slightly surpassed gram-negatives. However, most of these BSIs were related to the presence of a vascular catheter $[11,12]$. 
Table 3 Univariate and multivariate analysis of risk factors for death among 48 solid organ transplant recipients with bloodstream infection by gram positive bacteria

\begin{tabular}{|c|c|c|c|c|c|c|c|c|}
\hline \multirow[t]{3}{*}{ Variable } & \multicolumn{2}{|l|}{ Cases (48) } & \multicolumn{3}{|c|}{ Univariate } & \multicolumn{3}{|c|}{ Multivariate } \\
\hline & Death & Survival & $\mathrm{OR}$ & IC95 \% & $P$ & $\mathrm{OR}$ & IC95 \% & $p$ \\
\hline & $N(\%)$ & N (\%) & & & & & & \\
\hline Male gender & $10(34.5)$ & $19(65.5)$ & 0.90 & $0.27-3.02$ & 1.00 & & & \\
\hline ICU & $4(57.1)$ & $3(42.9)$ & 2.87 & $0.56-14.73$ & 0.23 & & & \\
\hline HAls & $10(52.6)$ & $9(47.4)$ & 3.49 & $1.01-12.05$ & 0.06 & & & \\
\hline Charlson $\geq 3$ & $5(29.4)$ & $12(70.6)$ & 0.66 & $0.18-2.35$ & 0.75 & & & \\
\hline Co-morb (liver disease) & 0 & 0 & & & & & & \\
\hline Co-morb (cancer) & $1(50.0)$ & $1(50.0)$ & 1.87 & $0.11-32.01$ & 1.00 & & & \\
\hline Co-morbidities $(\geq 2)$ & $1(20.0)$ & $4(80.0)$ & 0.42 & $0.04-4.11$ & 0.64 & & & \\
\hline Deceased donor & $11(47.8)$ & $12(52.2)$ & 2.90 & $0.85-9.93$ & 0.13 & & & \\
\hline Induction therapy & $17(77.3)$ & $5(22.7)$ & 0.60 & $0.47-0.77$ & 0.15 & & & \\
\hline Acute rejection (AR) & $9(47.4)$ & $10(52.6)$ & 2.36 & $0.70-7.95$ & 0.22 & & & \\
\hline AR-corticosteroids & $9(50.0)$ & $9(50.0)$ & 2.75 & $0.80-9.39$ & 0.13 & & & \\
\hline OB-MMF & $1(11.1)$ & $8(88.9)$ & 0.18 & $0.02-1.58$ & 0.13 & & & \\
\hline OB-Ciclosporine & $8(23.5)$ & $26(76.5)$ & 0.17 & $0.04-0.66$ & 0.02 & 0.20 & $0.03-1.19$ & 0.08 \\
\hline Double-J & $1(100.0)$ & $0(0)$ & 0.34 & $0.23-0.51$ & 0.35 & & & \\
\hline Pre tx dialysis & $16(39.0)$ & $25(61.0)$ & 3.84 & $0.42-34.94$ & 0.40 & & & \\
\hline CMV & $1(33.3)$ & $2(66.7)$ & 0.91 & $0.08-10.79$ & 1.00 & & & \\
\hline Shock & $6(85.7)$ & $1(14.3)$ & 16.4 & $1.76-151.7$ & 0.005 & 1.24 & $0.06-27.37$ & 0.89 \\
\hline Respiratory failure & $12(85.7)$ & $2(14.3)$ & 34.8 & $5.91-204.8$ & $<0.001$ & 28.3 & $2.53-317.1$ & 0.007 \\
\hline Pre tx diabetes & $2(33.3)$ & $4(66.7)$ & 0.90 & $0.15-5.50$ & 1.00 & & & \\
\hline Inadequate atb & $6(37.5)$ & $10(62.5)$ & 1.14 & $0.33-4.00$ & 1.00 & & & \\
\hline
\end{tabular}

ICU Intensive care unit, HAls Health-acquired infections, $O B$ onset of BSI, $T X$ transplant, atb antimicrobial

The urinary tract was the most commonly reported primary site of infection, having been identified in $36.4 \%$ of cases, which also supports the observation of a greater incidence of gram-negative infections in the study population. The presence of a double-J stent has also been shown to be related to gram-negative BSIs.

Among patients with GP BSI, the rate of acute rejection (39.6\%) was higher than in the GN group (20.4\%) $(p=0.008)$, which might help to explain the increased use of methylprednisolone in the first group (37.5 \% vs. $13.6 \%, p=0.003)$ and the higher mortality rate in the GP group (35.4\%).

The overall mortality rate in the study population (23.6\%) was very close to the rate observed in a population that included only kidney transplant recipients, from the same hospital and period (24.3\%) [13]. It is worth noting that the above mentioned Brazilian SCOPE study reports a mortality rate of $40 \%$, greater than that found in our study probably because it covers only nosocomial infections, where bacteria with reduced susceptibility are more frequently found [10].

In our study, we observed higher mortality among patients with gram-positive BSI (35.4\%) vs. gram-negative
BSI $(19.7 \%)(p=0.03)$. The mortality in the GN BSI group was lower than that reported in some other series (25-59 \%) [6, 7, 14]. On the other hand, GP BSI has been implicated as an independent risk factor for death among liver transplant recipients, with reported mortality rates of $28.5 \%$ for infections caused by $S$. aureus [15].

We found no association between antimicrobial adequacy and mortality, as has been described in the literature $[16,17]$. Most likely, the early adjustment of the antimicrobial therapy based on partial results from positive blood cultures may have contributed to this fact.

The presence of more than two comorbidities was independently associated with death in patients with GN BSI; the same was not observed in the GP group, where, in turn, there were more episodes of acute rejection and higher mortality rates, perhaps decreasing the relative impact of comorbidities in this group.

The need for mechanical ventilation as a risk factor for death, both in the GP and in the GN groups, was reported in earlier studies $[13,18,19]$ that assessed only kidney or liver transplant recipients, while our series includes other solid organ transplants. This was the only 
factor independently associated with increased mortality in our study, based on the multivariate analysis, regardless of the type of bacteria involved in the episode; perhaps this is a risk factor related to disease severity.

Another study that included only kidney transplant recipients identified septic shock, chronic renal failure requiring dialysis, comorbidities, and inadequate antimicrobial therapy as factors associated with increased mortality [20].

The limitations of our study are the retrospective analysis and the reliance on information based on clinical definitions and data from medical records, without direct contact with the patients. This limitation was minimized by the application of standards to ensure consistency in the analysis of the patients' medical records.

In this article we stress that GN and GP BSI in solid organ transplant recipients have different and important features. Understanding the characteristics and risk factors for death in the population of solid organ transplant recipients with bloodstream infections may help to design strategies to decrease mortality rates associated with this type of infection.

\section{Competing interests}

The authors declare that they have no competing interests.

\section{Authors' contributions}

Conceived and designed the experiments: TZSC, MSJ, ARM and LFAC. Analyzed the data: TZSC and ARM. Wrote the paper: TZSC, ARM and LFAC. All authors read and approved the final manuscript.

Received: 1 April 2015 Accepted: 13 July 2015

Published online: 01 October 2015

\section{References}

1. Tan K, Morissey P. Solid organ transplantation - overview. Med Health R I. 2007;90(3):72-5.

2. Medina-Pestana JO, Vaz MLS, Park SI. Organ transplant in Brazil. Transplant Proc. 2002;34:441-3.

3. Candel FJ, Grima M, Matesanz M, Cervera C, Soto G, Almela M, et al. Bacteremia and septic shock after solid-organ transplantation. Transplant Proc. 2005;37:4097-9.

4. McClean K, Kneteman N, Taylor G. Comparative risk of bloodstream infection in organ transplant recipients. Infect Control Hosp Epidemiol. 1994;15:582.

5. Moreno A, Mensa J, Almela M, Vilardell J, Navsa M, Claramont J, et al. 138 Episodes of bacteremia or fungemia in patients with solid organ (renal or hepatic) transplantation. Med Clin (Barc). 1994;103:161.

6. Wagener MM, Yu VL. Bacteremia in transplant recipients: a prospective study of demographics, etiologic agents, risk factors and outcomes. Am J Infect Control. 1992;20:239-47.

7. Singh N, Paterson DL, Gayowski T, Wagener MM, Marino IR. Predicting bacteremia and bacteremic mortality in liver transplant recipients. Liver Transpl. 2000;6:54.

8. Clinical and Laboratory Standards Institute (CLSI). Performance standards for antimicrobial susceptibility testing; eight informational supplement M2-A8. Wayne, PA: Clinical and Laboratory Standards Institute; 2005.

9. Bert F, Larroque B, Paugam-Burtz C, Janny S, Durand F, Dondero F, et al. Microbial epidemiology and outcome of bloodstream infections in liver transplant recipients: An analysis of 259 episodes. Liver Transplant. 2010;16:393-401.

10. Marra AR, Camargo LFA, Pignatari ACC, Sukiennik T, Behar PRP, Medeiros EAS, et al. Nosocomial bloodstream infections in brazillian hospitals: Analysis of 2,563 cases from a prospective nationwide surveillance study. J Clin Microbiol. 2011;49:1866-71.
11. Hsu J, Andes DR, Knasinski V, Pirsch J, Safdar N. Statins are associated with improved outcomes of bloodstream infection in solid-organ transplant recipients. Eur J Clin Microbiol Infect Dis. 2009:28:1343-51.

12. Torre-Cisneros J, Herrero C, Cañas E, Reguera JM, De La Mata M, Gómez-Bravo MA, et al. High mortality related with Staphylococcus aureus bacteremia after liver transplantation. Eur J Clin Microbiol Infect Dis. 2002;21:385-8.

13. Silva Jr M, Marra AR, Pereira CA, Medina-Pestana JO, Camargo LF. Bloodstream infection after kidney transplantation: epidemiology, microbiology, associated risk factors, and outcome. Transplanation. 2010;90(5):581-7.

14. Rodriguez C, Munoz P, Rodriguez-Creixems M, Yanez JF, Palomo J, Bouza E. Bloodstream infections among heart transplant recipients. Transplantation. 2006;81:384-91.

15. Bedini A, Codeluppi S, Cocchi S, Guaraldi G, Di Benedetto F, Venturelli C, et al. Gram-positive bloodstream infections in liver transplant recipients: Incidence, risk factors, and impact on survival. Transplant Proc. 2007;39:1947-9.

16. Palmer SM, Alexander BD, Sanders LL, Edwards $L$, Reller LB, Davis RD, et al. Significance of blood stream infection after lung transplantation: Analysis in 176 consecutive patients. Transplant. 2000;69:2360.

17. Silveira FP, Marcos A, Kwak EJ, Husain S, Shapiro R, Thai N, et al. Bloodstream infections in organ transplant recipients receiving alemtuzumab: No evidence of occurrence of organisms typically associated with profund T cell depletion. J Infect. 2006;53:241.

18. McHenry MC, Brawn HE, Popowiak KL, Banowksy LH, Deodhar SD. Septicemia in renal transplant recipients. Urol Clin North Am. 1976;3:647-65.

19. Korvick JA, Marsh W, Starzl TE, Yu VL. Pseudomonas aeruginosa bacteremia in patients undergoing liver transplantation: An emerging problem. Surgery. 1991;109:62-8.

20. Weinstein MP, Towns ML, Quartey SM, Mirrett S, Reimer LG, Parmigiani G, et al. The clinical of positive blood cultures in the 1990s: A prospective comprehensive evaluation of the microbiology, epidemiology, and outcome of bacteremia and fungemia in adults. Clin Infect Dis. 1997;24:584.

\section{Submit your next manuscript to BioMed Central and take full advantage of:}

- Convenient online submission

- Thorough peer review

- No space constraints or color figure charges

- Immediate publication on acceptance

- Inclusion in PubMed, CAS, Scopus and Google Scholar

- Research which is freely available for redistribution 